\title{
Interkulturelle Kommunikation
} und migrationssensible Hilfe

\section{Training von "critical incidents" in der beschäftigungsorientierten Beratung}

\author{
BETTINA FRANZKE UND \\ VITALIA SHVAIKOVSKA \\ Prof. Dr. Bettina Franzke ist Hoch- \\ schullehrerin für Interkulturelle \\ Kompetenzen und Diversity-Ma- \\ nagement an der Fachhochschu- \\ le für öffentliche Verwaltung \\ Nordrhein-Westfalen in Köln. Ihre \\ Arbeitsschwerpunkte sind interkul- \\ turelle Interaktionen im Kontext \\ Polizei, Kommunalverwaltung und \\ beschäftigungsorientierter Bera- \\ tung sowie Gendersensibilität in der \\ Beratung. \\ bettina.franzke@fhoev.nrw.de
}

Vitalia Shavaikovska ist Arbeitsvermittlerin im Arbeitgeberservice Jobcenter Dortmund. Im Jahre 2012 hat sie im Rahmen ihrer Bachelorthesis über critical incidents in der beschäftigungsorientierten Beratung geforscht. Im Sommer 2013 übernahm sie einen Lehrauftrag im Modul Grundlagen der Beratung an der Hochschule der Bundesagentur für Arbeit.

\author{
Viele hilfesuchende Menschen in Arbeitsagenturen \\ und Jobcentern weisen einen sogenannten \\ "Migrationshintergrund" auf. Bei deren Beratung \\ und Vermittlung können kulturelle Missverständnisse \\ oder gar Unverständnis ("critical incidents») bedeutsame \\ Hindernisse darstellen.
}

Situationen der Vielfalt und interkulturelle Herausforderungen lösen bei den Mitarbeiterinnen und Mitarbeitern, die in einem solchen beschäftigungsorientierten Beratungssetting agieren, nicht nur Freude, sondern oft auch Ängste, Unsicherheit und Befremden aus. Negativ bewertete Erfahrungen werden dabei in der Regel in direkten Zusammenhang mit der kulturellen Herkunft der Klientel gebracht. Einige Beratungs- und Vermittlungsfachkräfte versprechen sich durch die Teilnahme an einem interkulturellen Training Aufschluss darüber, wie bestimmte Denk- und Verhaltensweisen bei Menschen mit Migrationshintergrund zu erklären sind und was sie tun können (bzw. sollen), wenn sie sich als Akteurinnen und Akteure auf dem Arbeitsmarkt nicht respektiert, irritiert, verärgert über einfach nur überfordert fühlen. (1)

Interkulturelle Kompetenz gilt als ein Schlüssel zur Verständigung in interkulturellen Beratungssituationen. Die meisten Wirtschaftsunternehmen, die Fach- oder Führungskräfte ins Ausland entsenden, bereiten diese mit konkreten Handlungsempfehlungen auf ein bestimmtes Land oder einen bestimmten Kulturraum vor. Reine wissensvermittelnde Konzepte sind als Ausgangspunkt für das Handeln in einer Einwanderungsgesellschaft jedoch kaum brauchbar.

Ansätze zur Vermittlung von Handlungskompetenzen für den Umgang mit vielfältigen Menschen innerhalb einer Gesellschaft setzen nicht am Erwerb kulturspezifischen Wissens an. Vielmehr geht es zunächst um die Förderung von Sensibilität in interkulturellen Interaktionssituationen im Allgemeinen, die sich durch eine generelle Offenheit, Wertschätzung und Akzeptanz von Unterschieden auszeichnet.

Es handelt sich dabei um Eigenschaften, welche zu den Grundhaltungen der Beratung zählen und für die Entwicklung interkultureller Sensibilität einer besonderen Wahrnehmungsschärfung bedürfen. Am Beginn des interkulturellen Lernprozesses stehen das Erkennen der eigenen kulturellen Prägung (vgl. Krewer, 1994) und deren Auswirkungen auf die Beratung.

Zudem wird verdeutlicht, dass in den Settings, Verfahrensweisen und Begrifflichkeiten, die in den Institutionen am Arbeitsmarkt üblich sind, Kulturstandards zum Ausdruck kommen, die in Deutschland eine lange Tradition haben. So betonen "Eigenbemühungen « und "Zielvereinbarungen « beispielsweise die Orientierung am Einzelnen (Kulturstandard: Individualismus). Die konkrete Terminvereinbarung für Beratungsgespräche impliziert ein monochrones Zeitverständnis, das größten Wert auf minutengenaue Pünktlichkeit und die kommunikative Abwicklung von Themen ohne Zeitverzug legt. Und Büronummern, Informationsmaterial 
sowie Hinweisschilder ermöglichen eine räumliche Orientierung, die ohne verbale Kommunikation auskommt.

Neben kultureller Sensibilität ist es für Beratungsfachkräfte hilfreich, grundlegende Unterschiede zwischen Kulturen zu kennen. Letztlich geht es um die Aneignung von Handlungskompetenzen für Beratungssituationen. Diese ergeben sich unter anderem aus der Fähigkeit, genau zu beobachten (ohne zu bewerten), Mehrdeutigkeiten auszuhalten, die Perspektive wechseln zu können, Empathie zu zeigen, emotionale Betroffenheit zu kontrollieren, Achtsamkeit in der Kommunikation zu üben und Handlungsroutinen zu erweitern (vgl. Roth \& Köck, 2011).

Zentral ist die Entwicklung eines Gespürs und einer Analysefähigkeit dafür, was überhaupt kulturell bedingte Muster sind. Denn neben kulturellen Prägungen, können die (Lebens-) Situation und die Persönlichkeit als Erklärung von Denk- und Verhaltensweisen mit in Betracht gezogen werden. Nach Cohen-Emerique (2006) und Krewer (1994) steht am Zielpunkt interkulturellen Lernens kulturelle Kreativität, die es ermöglicht, neue, innovative Lösungen zu entwickeln.

Eine der effektivsten Trainingsmethoden zum Auf- und Ausbau von Handlungskompetenz - und das nicht nur in interkulturellen Kontext - ist die Critical-Incident-(CI)-Technik. Sie wurde 1954 von Flanagan zu eignungsdiagnostischen Zwecken entwickelt und wird bis heute als eine situationsbasierte Methode zur Arbeits- und Anforderungsanalyse eingesetzt (vgl. Schuler, 2006). Critical incidents in der Personalauswahl sind Situationen, in denen

- in denen die handelnden Personen auf Barrieren stoßen, ihnen etwas widerfährt und sie sich wahrscheinlich missverstehen,

- die sie als konfliktträchtig, irritierend, frustrierend, schwierig, merkwürdig oder rätselhaft erleben,

- die anderes oder neu interpretiert werden, wenn die Personen über kulturelles Wissen verfügen (vgl. Fiedler et al., p. 97; Thomas, S. 415).

Mit diesem Verständnis von critical incidents verbindet sich der »Kulturassimilator «. Dieser ist Bestandteil von (Selbst-) Lernprogrammen, die auf spezifische Kulturen bezogenen sind und in denen die Lernenden schriftlich mit einem bestimmten Szenario konfrontiert werden und anschließend aus vier Antwortoptionen die wahrscheinlichste kulturelle Erklärung herausfinden sollen. In der von Thomas herausgegebenen Reihe "Beruflich in ... « sind inzwischen rund 30 Bände erschienen, die nach dem Kulturassimilator aufgebaut sind.

Kulturassimilatoren sind allerdings insofern kritisch zu bewerten, als dass sie die Gefahr von Stereotypisierung in sich bergen: Es wird angenommen, dass sich Menschen aus einem bestimmten Land relativ ähnlich verhalten, was de facto bei Menschen mit Migrationshintergrund jedoch nicht der Fall sein muss.

Um die Critical-Incidents Technik für die interkulturelle Verständigung in der beschäftigungsorientierte Beratung nutzbar zu machen, bieten sich die von Grosch und Groß (2005) sowie Wight (1995) konzipierten Weiterentwicklungen an: Hier werden die Lernenden mit einer komplexen migrationsspezifischen Fragestellung konfrontiert, die

\section{"Putzen ist auch okay"}

bestimmte Verhaltensweisen als besonders erfolgreich oder nicht erfolgreich im Hinblick auf ein bestimmtes Ziel dienen und die eine Unterscheidung zwischen geeigneten und nicht geeigneten Personen erlauben.

Fiedler, Mitchell und Triandis (1971) sowie Thomas (1993) haben die CI-Technik auf interkulturelle Interaktionen übertragen und verstehen unter critical incidents Situationen, nicht auf einen einzelnen Kulturraum festgelegt, sondern der Alltagswelt vielfältiger Gesellschaften entnommen ist. Das Szenario soll entlang bestimmter, offen gehaltener Leitfragen bearbeitet werden, welche das eigene Erleben und die Irritation eigener Standards sichtbar machen, und darüber hinaus zur Perspektiverweiterung anregen und letztlich zur Entwicklung von Handlungsstrategien auffordern.
Die Konstruktion von critical incidents im Sinne von Grosch und Groß (2005, S. 246 f.) sowie Wight (1993, p. 130) umfasst sechs Schritte (vgl. Kasten "Wie man critical incidents konstruieren kann«). Es empfiehlt sich, pro Fall vier bis sechs Fragen zu auszuwählen, die für das interkulturelle Lernen besonders passend sind (zur fragegestützten Analyse interkultureller Situationen in der sozialen Arbeit siehe auch CohenEmerique, 2006).

Um interkulturelle Trainings in der Aus- und Weiterbildung von Beratungsfachkräften zu unterstützen, haben die Autorinnen dieses Beitrags zum einen jeweils zehn critical incidents über teilnehmende Beobachtungen in Agenturen für Arbeit sowie Jobcentern erhoben, zum anderen wurden weitere neun critical incidents in Seminaren oder Gesprächen mit Beratungsfachkräften in Jobcentern erfragt und dort erarbeitet. (2)

Die empirische Erhebung von critical incidents in der beschäftigungsorientierten Beratung hat gezeigt, dass die klassischen, von Hofstede (2011) benannten Kulturunterschiede, zur Interpretation interkultureller Interaktionen im beschäftigungsorientierten Kontext nur selten in Betracht kommen. Wie die Feststellung einer als Reiningungskraft in Deutschland arbeitenden polnischen Diplomphysikerin verdeutlicht - »Putzen ist auch okay « -, sind es oft Aspekte wie die subjektive Verarbeitung von Erfahrungen auf dem deutschen Arbeitsmarkt, individuelle Überzeugungen über die Chancen und Grenzen einer Teilhabe, erprobte Erfolgsstrategien zum Überleben in der deutschen Gesellschaft, das Erleben von Fremdheit, die Lebenssituation, Fremdzuschreibungen sowie Ergebnisse komplexer Identitätskonstruktionen, welche das Denken und Handeln einer Person bestimmen.

Um die gesellschaftliche Teilhabe von Menschen mit Migrationshintergrund zu stärken und die interkulturelle Kompetenz der Mitarbeiterinnen und Mitarbeiter zu fördern, streben viele Institutionen beschäftigungsorientierter Beratung nach interkultureller Öffnung. Die Agenturen für Arbeit und Jobcenter wollen also vielfältiger werden und den Anteil von Menschen mit Migrationshintergrund unter den eigenen Beschäftigten erhöhen (vgl. z. B. Behrens, 2011). 


\section{Chancen und Gefahren}

Methodisch gesehen machen sich die Teilnehmenden in interkulturellen Trainings nach dem Vorstellen eines critical incidents zunächst in Einzelarbeit Notizen zu dem jeweiligen Fall. Nach fünf Minuten kann zu einem Austausch in einer Kleingruppe (3 bis 5 Personen) übergegangen werden, in der verschiedene Perspektiven zusammengetragen und diskutiert werden. Anschließend werden die Ergebnisse der Fallanalyse in der Gesamtgruppe vorgestellt und es werden Bezüge zu den theoretischen Konzepten oder Erfahrungswerten Anderer in der Gruppe hergestellt (vgl. Wight, 1995, p. 131 ff.). In der Großgruppe können mehrere critical incidents parallel bearbeitet werden. Die Teilnehmenden können auch selbst critical incidents formulieren, wobei sie gegebenenfalls Anleitung und Hilfestellung durch die Trainerin oder den Trainer bedürfen. Für die Bearbeitung eines critical incidents entlang dieser drei Phasen (Einzelarbeit, Kleingruppenarbeit, Präsentation in der Großgruppe) sollten 30 bis 45 Minuten eingeplant werden.

Critical incidents beinhalten großes Lernpotenzial, sie fördern den Perspektivwechsel und die Persönlichkeitsentwicklung im interkulturellen und migrationsspezifischen Kontext. Layes (2007) bezeichnet critical incidents als »mächtigste Methode (...) zur Initiierung interkultureller Lernprozesse" ( $S$. 386). Positiv hervorzuheben ist, dass sie sehr praxis- und realitätsnah sind, auf bestimmte Zielgruppen und Berufsfelder abgestimmt werden können (vgl. Grosch \& Groß, 2005, S. 247) sowie Handlungskompetenz weiterentwickeln, ohne dabei die Betroffenheit der Lernenden und deren Wahrnehmungsschärfung außer Acht zu lassen. Die Szenarien sprechen die Lernenden gleichermaßen kognitiv und emotional an, aktivieren die von den Akteurinnen und Akteuren der beschäftigungsorientier- ten Beratung oft vermisste Kreativität und bilden letztlich eine den Menschen vertraute Methode: Geschichten erzählen ist eine uralte Form der Wissensvermittlung, die auch in der informellen Aufarbeitung belastender Erfahrungen im kollegialen Umfeld einen hohen Stellenwert einnimmt.

In den Chancen von critical incidents liegen zugleich deren Grenzen: Die Szenarien sind oft sehr situationsspezifisch angelegt und können nur bedingt auf andere Situationen übertragen werden (vgl. Grosch \& Groß, 2005, S. 248 f.). Auch dann, wenn kulturelle Herkunft und Beschäftigungssituation ähnlich sind, kann eine andere Person in ihrer Persönlichkeit, ihren Vorhaben und ihrer kultureller Identität ganz anders sein als die Hauptfigur im critical incident.

\section{Resümee}

Interkulturelle Sensibilität bedeutet, nicht sofort Lösungsmuster parat zu haben,

\section{Wie man critical incidents konstruieren kann}

Das sechs Schritte umfassende Vorgehen bei der Konstruktion eines critical incidents im Sinne von Grosch und Groß (2005, S. 246f.) sowie Wight (1993, p. 130) ist nachfolgend näher beschrieben. Es empfiehlt sich, pro Fall vier bis sechs Fragen zu formulieren, die für das interkulturelle Lernen besonders passend sind (zur fragegestützten Analyse interkultureller Situationen in der sozialen Arbeit s. auch Cohen-Emerique, 2006).

\section{Erster Schritt: Ideen finden}

- Anknüpfungspunkte für critical incidents: eigener Wissens- und Erfahrungsschatz, Erfahrungsberichte von Anderen, Medienreportagen, Dokumentationen, Filme, Werbung, Publikationen zur interkulturellen Kommunikation

- Anforderungen an critical incidents: praxis- und realitätsnah, dicht am Berufsfeld der Zielgruppe, durch Beobachtung/ Expertengespräche gewonnen, bringen migrationsspezifische Themen zur Sprache, die grundlegend und relevant für viele Interaktionen sind

\section{Zweiter Schritt: Thema oder Problem formulieren}

- Wahl des Formats (Kombinationen möglich)

1. Kulturdialog: Ereignisse in wörtlicher Rede

2. Kurze Fallbeschreibungen, Situationsskizzen

3. Konfliktszenario: Wiedergabe eines Konfliktes

4. Weiteres Material: Aktenvermerk, Zeitungsausschnitt usw.

- Beschreibung der Situation und Formulierung des Titels

- 3 bis 5: Personen, Kontext und Handlungen beschreiben

\section{Dritter Schritt: Handelnde Personen festlegen}

- maximal drei, vier Personen, Alter, Beruf, Geschlecht, Hintergrund, Namen

\section{Vierter Schritt: Kontext beschreiben}

n Ort und Zeit, Skizze der Umstände

\section{Fünfter Schritt: Handlungen beschreiben}

- Was sagen und tun die beteiligten Personen?

\section{Sechster Schritt: Fragestellungen formulieren}

A. Überprüfung der Geschehnisse:

Was genau ist passiert? Was ist das Problem?

B. Eigenes Erleben und eigene Sichtweisen

- Was sind meine ersten Gedanken? Habe ich schon einmal eine ähnliche Situation beobachtet oder selbst erlebt?

- Was empfinde ich (z. B. Angst, Ekel, Empörung, Ablehnung)? Was genau schockt, irritiert oder stört mich?

- Welche meiner Werte und Grundsätze sind erschüttert worden?

- Welche eigenkulturellen Standards sind berührt?

C. Entwicklung von Interpretationen

- Was denkt, was fühlt Person $\mathrm{XY}$ ?

- Was weiß ich von der anderen Person? Was ist ihr Bezugssystem?

- Hypothesenbildung: Welche Gründe kann Person XY haben, so zu handeln? Versuchen Sie, mehrere Interpretationsmöglichkeiten zu finden: kulturell, personenspezifisch, sozial, kontextbezogen, institutionell, migrationsspezifisch

D. Perspektivwechsel und Empathie

- Was vermute ich, wie die andere Person die Situation erlebt?

- Welcher Handlungsspielraum steht Person XY zur Verfügung?

- Was verstehe ich nicht?

E. Entwicklung von Handlungsstrategien

- Was will ich erreichen?

- Welche Fragen möchte ich stellen?

- Was kann eine neue Norm sein, ein gemeinsames Feld ("Zwischenraum «), in dem jeder seine Identität bewahrt und gleichzeitig den Weg des Anderen betritt?

- Was sind konkrete Schritte? Was würde ich Person XY empfehlen? 
sondern vielmehr sich Zeit zu nehmen, genauer hinzuschauen, mehr verstehen $\mathrm{zu}$ wollen als bisher und sich in einen Aushandlungsprozess hineinzubegeben.

Das Ziel ist dabei, ein tragfähiges Arbeitsbündnis aufzubauen und nachhaltige Lösungen zu entwickeln. Dieser Prozess erfordert einerseits Geduld, andererseits führt er zu mehr Zufriedenheit bei den Beschäftigten, da sie mehr Informationen gewinnen, passgenauere Strategien entwickeln können und auf größere Kooperationsbereitschaft treffen.

Wünschenswert ist, dass interkulturelle Trainings fester Bestandteil eines Qualifizierungskonzeptes aller Institutionen beschäftigungsorientierter Beratung werden, der Blick für Beratungen im interkulturellen Kontext geschärft wird, Beratungen im interkulturellen Kontext mehr Zeit eingeräumt wird und darüber hinaus die interkulturelle Öffnung der Institutionen selber weiter vorangebracht wird.

\section{Anmerkungen}

(1) Wir danken Anja Taubner und Alessia Gordienko für die kritische Durchsicht und guten Impulse zu einer vorhergehenden Fassung dieses Beitrags.

(2) Die Gesamtheit aller critical incidents, erweitert um solche für interkulturelle Interaktionen in der kommunalen Verwaltung sowie bei der Polizei, werden von den Autorinnen gerade für eine Buchveröffentlichung aufbereitet.

\section{Literatur}

Behrens, B. (2011). Demographiesensibles Personal- und Diversity Management. Bei der Bundesagentur für Arbeit (BA). www.chartader-vielfalt.de/service/publikationen/ jung-alt-bunt/gute-praxis-im-oeffentlichensektor-und-im-sozialen-bereich/ demographiesensibles-personal-und-diversitymanagement.html (Abruf am 07.01.2014).

Cohen-Emerique, M. (2006). Der Kulturschock als Weiterbildungsmethode und als Forschungsinstrument. In H. Nicklas, B. Müller \& H. Kordes (Hrsg.), Interkulturell denken und handeln. Theoretische Grundlagen und gesellschaftliche Praxis (S. 317-327). Bonn: Bundeszentrale für politische Bildung.

Fiedler, F. E., Mitchell, T., \& Triandis, H. C. (1971). The culture assimilator: An approach to cross-cultural training. Journal of Applied Psychology, 55, 95-102.

Flanagan, John C. (1954). The Critical Incident Technique. Psychological Bulletin, 51, Vol. 4, 327-358. www.analytictech.com/mb870/ Readings/flanagan.pdf (Abruf am 07.01.2014). Grosch, H. \& Groß, A. (2005). Entwicklung spezifischer Vermittlungsformen und Medien. In W. R. Leenen (Hg.), Bausteine zur interkulturellen Qualifizierung der Polizei (S. 227-271). Münster usw.: Waxmann.

Hofstede, G. (2011). Lokales Denken, globales Handeln (5. Aufl.). München: C. H. Beck. Siehe auch: www.geert-hofstede.com.

Kohn, K. (2011). Migrationsspezifische beschäftigungsorientierte Beratung - spezifische Themen, spezifische Bedarfe. Ergebnisse einer Delphi-Breitband-Erhebung. Berlin: Facharbeitskreis "Beratung" vom Netzwerk "Integration durch Qualifizierung«.www.kohnpage.de/ Text2011a.pdf (Abruf am 07.01.2014).

Krewer, B. (1994). Interkulturelle Trainingsprogramme - Bestandsaufnahme und Perspektiven. In H.-G. Arzt (Hg.), Europäische Qualifikation durch deutsch-französische Ausbildung? Die Bedeutung der Unterschiede nationaler Bildungssysteme für die internationale wirtschaftliche Zusammenarbeit (S. 36-50). Ludwigsburg: Deutsch-Französisches Institut. Layes, G. (2007). Kritische Interaktionssituation. In J. Straub, A. Weidemann \& D. Weidemann (Hg.), Handbuch interkulturelle Kommunikation und Kompetenz. Grundbegriffe - Theorien Anwendungsfelder (S. 384-391). Stuttgart,

\section{Weimar: Metzler}

Roth, J. \& Köck, C. (Hrsg.) (2011). Interkulturelle Kompetenz - Culture Communication Skills. Handbuch für die Erwachsenenbildung (2. Aufl.) München: Bayerischer Volkshochschulverband.

Schuler, H. (2006). Lehrbuch der Personalpsychologie (2. Aufl.). Göttingen: Hogrefe.

Thomas, A. (Hrsg.). Beruflich in ... (verschiedene Länder). Trainingsprogramm für Manager, Fachund Führungskräfte.

Thomas, A. (1993). Psychologie interkulturellen Lernens und Handelns. In A. Thomas (Hg.), Kulturvergleichende Psychologie. Eine Einführung (S. 377-424). Göttingen: Hogrefe.

Wight, A. (1995). The Critical Incident as a Training Tool. In S. Fowler

\& M. Mumford (eds.), Intercultural Sourcebook Vol.1. Cross-Cultural Training Methods (pp. 127-140). Yarmouth: Intercultural Press.

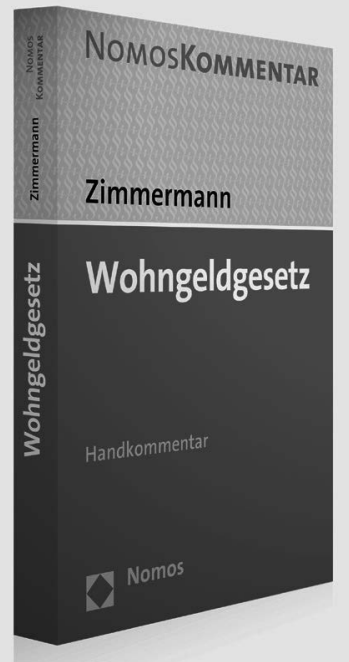

\section{Wohngeldgesetz \\ Handkommentar \\ Von RA Ludwig Zimmermann, FASOzR u FAArbR \\ 2014, 189 S., brosch., 44,- $€$, ISBN 978-3-8329-4836-8}

Der neue Handkommentar behandelt das aktuelle Wohngeldrecht in allen Facetten und zeigt Lösungswege für häufige Fragen in der alltäglichen Beratungsund Behördenpraxis auf wie

- alle relevanten Auslegungsfragen zu den gesetzlich verankerten Leistungsansprüchen, deren Grenzen wie Höhe

- die Abgrenzung zu den Leistungen der Grundsicherung

- das bei der Rechtsanwendung zu beachtende Verwaltungsverfahren

- die Überschneidungen zum Ausländer- und Europarecht

- die Besonderheiten der Einkommensermittlung insbesondere im Hinblick auf die Abgrenzung zu den Transferleistungen.

www.nomos-shop.de/11660 